Journal for ImmunoTherapy of Cancer

\section{Baseline prognostic nutritional index and changes in pretreatment body mass index associate with immunotherapy response in patients with advanced cancer}

To cite: Johannet P, Sawyers A, Qian Y, et al. Baseline prognostic nutritional index and changes in pretreatment body mass index associate with immunotherapy response in patients with advanced cancer. Journal for ImmunoTherapy of Cancer 2020;8:e001674. doi:10.1136/jitc-2020-001674

- Additional material is published online only. To view, please visit the journal online (http://dx.doi.org/10.1136/jitc2020-001674).

Accepted 20 October 2020

Check for updates

(c) Author(s) (or their employer(s)) 2021. Re-use permitted under CC BY-NC. No commercial re-use. See rights and permissions. Published by BMJ.

${ }^{1}$ Medicine, New York University School of Medicine, New York City, New York, USA ${ }^{2}$ Dermatology, New York University School of Medicine, New York City, New York, USA ${ }^{3}$ Population Health, New York University School of Medicine, New York City, New York, USA

Correspondence to

Dr Iman Osman;

iman.osman@nyulangone.org

\section{ABSTRACT}

Background Recent research suggests that baseline body mass index (BMI) is associated with response to immunotherapy. In this study, we test the hypothesis that worsening nutritional status prior to the start of immunotherapy, rather than baseline BMI, negatively impacts immunotherapy response.

Methods We studied 629 patients with advanced cancer who received immune checkpoint blockade at New York University. Patients had melanoma $(n=268)$, lung cancer $(n=128)$ or other primary malignancies $(n=233)$. We tested the association between BMl changes prior to the start of treatment, baseline prognostic nutritional index (PNI), baseline BMI category and multiple clinical end points including best overall response (BOR), objective response rate (ORR), disease control rate (DCR), progression-free survival (PFS) and overall survival (OS).

Results Decreasing pretreatment $\mathrm{BMI}$ and low PNI were associated with worse BOR ( $p=0.04$ and $p=0.0004)$, ORR $(p=0.01$ and $p=0.0005)$, DCR $(p=0.01$ and $p<0.0001)$, PFS $(p=0.02$ and $p=0.01)$ and $0 S(p<0.001$ and $p<0.001)$. Baseline BMI category was not significantly associated with any treatment outcomes.

Conclusion Standard of care measures of worsening nutritional status more accurately associate with immunotherapy outcomes than static measurements of BMI. Future studies should focus on determining whether optimizing pretreatment nutritional status, a modifiable variable, leads to improvement in immunotherapy response.

\section{BACKGROUND}

Immune checkpoint inhibitors (ICIs) produce durable clinical response for a subset of patients with cancer, but many individuals do not benefit, and many more develop autoimmune toxicity. ${ }^{1-4}$ Predictive markers are needed to optimize patient selection for treatment. Several studies suggest a link between baseline body mass index (BMI) and response to ICIs. ${ }^{5-9}$ Obesity predisposes to immune system dysfunction, which means that it could simultaneously represent an opportunity to reinvigorate the anticancer immune response through checkpoint blockade. ${ }^{10}$ However, there are substantial discordances in the data including variation in the magnitude and direction of the reported associations. In fact, we recently reported heterogenous trends in the relationship between baseline BMI and ICI response in melanoma. ${ }^{11}$ Specifically, we found that overweight and obese patients receiving combination immunotherapy had a significant progression-free survival (PFS) benefit, but there was no difference in PFS in those who received anti-programmed cell death protein 1 or anti-cytotoxic T-lymphocyteassociated protein 4 monotherapy. Moreover, there was no overall survival (OS) benefit seen in overweight patients or patients with obesity.

One limitation to most of the extant literature is that BMI was evaluated at a single point in time. Static assessments fail to capture dynamic changes in weight and nutrition. Some patients may have a lower BMI in the setting of worsening disease burden. In this case, reverse causation bias could lead to a distorted interpretation of the data whereby obesity is misconstrued as protective, when in fact it is declining nutritional status and concomitant systemic inflammation that dampen ICI response. BMI reflects nutritional status, but we postulate that pretreatment BMI changes prior to the start of treatment provide a more comprehensive picture than baseline values taken at the time of treatment initiation. In this study, we hypothesized that worsening of nutritional status before the start of immunotherapy, rather than baseline BMI, might negatively 
impact immunotherapy response. To test this hypothesis, we investigated the association between pretreatment BMI trends in the month before treatment initiation, baseline BMI category and immunotherapy response in a cohort of patients with advanced cancer. Given that nutritional status can also be assessed using serum laboratory values, we concurrently investigated the predictive potential of the baseline prognostic nutritional index (PNI), which is a validated tool with established prognostic utility in patients with cancer. ${ }^{12-17}$

\section{METHODS}

\section{Patient population}

The final study cohort consisted of patients with stage III or IV cancer who were treated between 2012 and 2020 with atezolizumab, avelumab, durvalumab, ipilimumab, nivolumab, pembrolizumab or tremelimumab as monotherapy or combination therapy. To be included, patients needed height and weight measurements at the time of treatment initiation (baseline value) and at a pretreatment visit 15-45 days before treatment start. In addition, patients needed baseline serum albumin and absolute lymphocyte counts. Patients were allowed in this study whether they received ICIs alone or in combination with other agents such as chemotherapies. Exclusion criteria included stage II disease, adjuvant checkpoint blockade and BMI $<18.5$ at either the pretreatment or baseline assessment.

\section{Assessment of BMI and PNI}

BMI was calculated as weight in kilograms divided by height in meters squared. BMI was classified as normal (18.5-24.9), overweight (25-29.9) and obese ( $\geq 30)$. For a separate analysis, we also classified baseline BMI category as normal $(<25)$ versus elevated $(\geq 25)$. To calculate the pretreatment BMI trend, we subtracted the baseline BMI from the pretreatment BMI. Patients were then divided into two categories: those whose pretreatment BMI decreased (pretreatment BMI-baseline BMI $>0$ ) and those whose BMI did not decrease (pretreatment BMIbaseline BMI $\leq 0$ ). We also evaluated pretreatment BMI trends as a continuous variable. Since a minor decrease in weight could be due to differences in measurements techniques rather than true clinical change, we performed a subset analysis of patients whose BMI decreased by $\geq 2 \%$. The prognostic nutritional index was calculated as previously reported: PNI $=(10 \times a$ albumin $(\mathrm{g} / \mathrm{dL}))+(0.005 \times \mathrm{ab}-$ solute lymphocyte count (per $\left.\mathrm{mm}^{3}\right)$ ). We used an established cut-off score of 45 to divide patients into two groups: low PNI $(<45)$ versus normal PNI $(\geq 45) \cdot{ }^{12} 1318$ As a quality control measure, we generated receiver operating characteristic curves and calculated the optimal threshold for our own study cohort using Youden's index. The optimal cut-off was $\mathrm{PNI}=44.25$ for $\mathrm{PFS}$ and $\mathrm{PNI}=44.75$ for OS, thus validating our decision to use the previously established cut-off score of 45 in our analyses. Finally, we performed subset analyses excluding patients who met criteria for cachexia or refractory cachexia, which were defined as previously reported with the caveat that we did not have BMI data from 6 months before treatment initiation. ${ }^{19}$ Patients with weight loss $>5 \%$ or with a pretreatment BMI $<20$ and any degree of weight loss $>2 \%$ were classified as cachectic, as were patients with an Eastern Cooperative Oncology Group (ECOG) performance status of 3 .

\section{Clinical outcomes}

We evaluated the relationship between baseline BMI category, pretreatment BMI trend and PNI with the following clinical outcomes: best overall response (BOR), objective response rate (ORR), disease control rate (DCR), PFS and OS. Response was classified as complete response (CR), partial response (PR), stable disease (SD) or progressive disease (PD) according to the revised Response Evaluation Criteria in Solid Tumors guideline V.1.1. ORR was the proportion of patients with CR or PR. DCR was the proportion of patients with CR, PR or SD. PFS was the time from treatment start until disease progression or death from cancer. ${ }^{20}$ OS was the time from treatment start until death from any cause.

\section{Statistical analyses}

Associations with BOR, ORR and DCR were assessed using the chi-square test. The 95\% CIs were calculated using the Clopper-Pearson method. We generated Kaplan-Meier survival curves to determine the association between PFS or OS and baseline BMI category, pretreatment BMI trend and baseline PNI category. We used the log-rank test for univariable analyses. We used multivariable Cox proportional hazards models to analyze the association between PFS and OS with baseline BMI category, pretreatment BMI trend and PNI. The multivariable analyses were stratified by cancer type and adjusted for age, sex, disease stage, ECOG performance status, treatment regimen, treatment line and whether immunotherapy was given alone or with other therapy. All analyses were performed using R V.3.6.1. Statistical tests were two-sided and $p<0.05$ was considered statistically significant.

\section{RESULTS \\ Patient characteristics}

A total of 629 patients met inclusion criteria for this study (online supplemental figure 1). Table 1 shows their clinical and demographic characteristics. The majority had melanoma $(n=268)$ or lung cancer $(n=128)$. The remaining patients had kidney $(\mathrm{n}=37)$, breast $(\mathrm{n}=36)$, head and neck $(n=25)$, liver $(n=25)$, genitourinary $(n=21)$, brain $(n=18)$, soft tissue $(n=18)$, non-melanoma skin $(n=14)$, gastric $(n=13)$, ovarian $(n=11)$, pancreatic $(n=9)$ or uterine cancer $(n=6)$. In total, 308 patients $(49.0 \%)$ had a decrease in pretreatment BMI and 321 (51.0\%) patients had no decrease in pretreatment BMI. There were 193 (30.7\%) patients with a low PNI and 436 (69.3\%) patients 
Table 1 Clinical and demographic characteristics of the cohort

\begin{tabular}{|c|c|}
\hline & $\mathbf{N}(\%)$ \\
\hline Total number of patients & 629 \\
\hline \multicolumn{2}{|l|}{ Age (years) } \\
\hline Mean & 63.0 \\
\hline Range & 20.4-94.6 \\
\hline \multicolumn{2}{|l|}{ Sex } \\
\hline Female & $284(45.2)$ \\
\hline Male & $345(54.8)$ \\
\hline \multicolumn{2}{|l|}{ ECOG } \\
\hline 0 & $300(47.7)$ \\
\hline 1 & $275(43.7)$ \\
\hline$\geq 2$ & $54(8.6)$ \\
\hline \multicolumn{2}{|l|}{ Primary tumor } \\
\hline Melanoma & $268(42.6)$ \\
\hline Lung & $128(20.3)$ \\
\hline Other & $233(37.0)$ \\
\hline \multicolumn{2}{|l|}{ Stage } \\
\hline III & $86(13.7)$ \\
\hline IV & $543(86.3)$ \\
\hline \multicolumn{2}{|l|}{ Treatment regimen } \\
\hline Anti-CTLA-4 & 66 (10.5) \\
\hline Anti-PD-1 or Anti-PD-L1 & $415(66.0)$ \\
\hline Anti-PD-1+anti-CTLA-4 & $148(23.5)$ \\
\hline \multicolumn{2}{|l|}{ Treatment line } \\
\hline First & $448(71.2)$ \\
\hline Non-first & $181(28.8)$ \\
\hline
\end{tabular}

Immunotherapy alone or in combination with other therapy

\begin{tabular}{|cc|}
\hline Alone & $447(71.2)$ \\
\hline With other therapy & $182(28.9)$ \\
\hline Pretreatment BMI trend & \\
\hline Decreasing & $308(49.0)$ \\
\hline Not decreasing & $321(51.0)$ \\
\hline Baseline prognostic nutritional index & \\
\hline Low (<45) & $193(30.7)$ \\
\hline Normal $\geq 45)$ & $436(69.3)$ \\
\hline Alive status & \\
\hline Alive & $304(48.3)$ \\
\hline Deceased & $325(51.7)$ \\
\hline Cancer-specific death & $296(91.1)$ \\
\hline Non-cancer-related death & $29(8.9)$ \\
\hline Median follow-up (months) & 21.7 \\
\hline
\end{tabular}

BMI, body mass index; CTLA-4, cytotoxic T-lymphocyteassociated protein 4; ECOG, Eastern Cooperative Oncology Group; PD-1, programmed cell death protein 1; PD-L1, programmed death-ligand 1. with a normal PNI. There were 56 patients who met one or more of the criteria for cachexia.

\section{Association of pretreatment BMI trends with immunotherapy response}

Compared with patients whose pretreatment BMI did not decrease, those with any decrease experienced worse ORR (27.6\% (95\% CI 22.7 to 33.0$)$ vs $38.0 \%$ (95\% CI 32.7 to 43.6$) ; \mathrm{p}=0.01)$ and worse DCR $(45.8 \%$ (95\% CI 40.1 to 51.5 ) vs $56.1 \%$ (95\% CI 50.1 to 61.6 ); $\mathrm{p}=0.01$ ) (table 2). In total, 145 patients had a decrease in BMI $\geq 2 \%$. These individuals had significantly worse ORR and DCR compared with those with a decrease in BMI $<2 \%$ or no decrease at all ( $\mathrm{p}=0.02$ for ORR; $\mathrm{p}=0.003$ for DCR). After excluding patients who met criteria for cachexia, the association between decreasing BMI and worse ORR and DCR remained significant $(\mathrm{p}=0.004$ and $\mathrm{p}=0.03$ ).

In univariable analyses, PFS was worse for patients with any decrease in BMI $(p=0.03)$, for patients with $\geq 2 \%$ decrease in BMI $(p=0.004)$ and for non-cachectic patients with any decrease in BMI $(p=0.03)$ (figure 1$)$. These associations remained significant in multivariable analyses. Patients whose BMI decreased prior to treatment were more likely to suffer worse PFS (HR 1.27; 95\% CI 1.05 to $1.54 ; \mathrm{p}=0.02$ ), as were patients with $\geq 2 \%$ decrease in BMI (HR 1.27; 95\% CI 1.01 to $1.59 ; \mathrm{p}=0.04$ ), and noncachectic patients with any decrease (HR 1.29; $95 \%$ CI 1.06 to $1.58 ; \mathrm{p}=0.01)$. The association between pretreatment BMI and PFS remained significant when analyzing BMI trend as a continuous variable $(\mathrm{p}=0.01)$ (table 3$)$.

In univariable analyses, OS was worse in patients with any decrease in BMI $(\mathrm{p}=0.0003)$, in patients with a decrease in BMI by $\geq 2 \%(p<0.0001)$ and in non-cachectic patients with any decrease in BMI $(p=0.002)$ (figure 1$)$. These associations remained significant in multivariable analyses. Patients with any decrease in BMI had worse OS (HR 1.61; 95\% CI 1.27 to 2.05; $\mathrm{p}<0.001$ ) as did patients with $\geq 2 \%$ decrease in BMI (HR 1.56; 95\% CI 1.21 to 2.00; $\mathrm{p}=0.001)$ and non-cachectic patients with a decrease in BMI (HR 1.62; 95\% CI 1.26 to 2.08; $\mathrm{p}<0.001$ ). The association between decrease in pretreatment BMI and worse OS was also significant when analyzing BMI trends as a continuous variable (HR 1.19; CI 1.08 to $1.32 ; \mathrm{p}=0.001$ ) (table 3).

\section{Association of baseline PNI with immunotherapy response}

Patients with a low PNI had worse ORR $22.8 \%$ (95\% CI 17.1 to 29.4 ) vs $37.4 \%$ (95\% CI 32.8 to 42.1 ); $\mathrm{p}=0.0005$ ) and worse DCR ( $37.8 \%$ (95\% CI 31.0 to 45.1 ) vs $56.9 \%$ (95\% CI 52.1 to 61.6$)$; $\mathrm{p}<0.0001$ ) (table 4 ). These associations remained significant after excluding patients who met criteria for cachexia $(p=0.001$ for ORR and $\mathrm{p}=0.0002$ for DCR). In univariable analyses, patients with low PNI had significantly worse PFS $(\mathrm{p}<0.0001)$ and OS $(p<0.0001)$ than those with normal PNI (figure 2). Similar trends were seen after excluding patients who met criteria for cachexia ( $p=0.0002$ for PFS and $p<0.0001$ for OS). In multivariable analyses, lower PNI was associated 
Table 2 Response to immunotherapy based on pretreatment BMI trend

\begin{tabular}{|c|c|c|c|}
\hline Outcome & Any decrease $(n=308)$ & No decrease $(n=321)$ & $P$ value \\
\hline Best overall response-no. (\%) & & & 0.04 \\
\hline Complete response (CR) & $31(10.1)$ & $37(11.5)$ & \\
\hline Stable disease (SD) & $56(18.2)$ & $58(18.1)$ & \\
\hline Progressive disease (PD) & $162(52.6)$ & $135(42.1)$ & \\
\hline Objective response rate* & & & 0.01 \\
\hline No. (\%) & $85(27.6)$ & $122(38.0)$ & \\
\hline $95 \% \mathrm{Cl} \dagger$ & 22.7 to 33.0 & 32.7 to 43.6 & \\
\hline Disease control rateł & & & 0.01 \\
\hline No. (\%) & $141(45.8)$ & $180(56.1)$ & \\
\hline
\end{tabular}

${ }^{*}$ Objective response rate was defined as the percentage of patients who had CR or PR. †The $95 \%$ Cls were calculated using the Clopper-Pearson method.

$\ddagger$ Disease control rate was defined as the percentage of patients who had CR, PR or SD

with significantly worse PFS (HR $1.34 ; 95 \%$ CI 1.06 to $1.69 ; \mathrm{p}=0.01$ ) and OS (HR $1.65 ; 95 \%$ CI 1.27 to 2.13 ; $\mathrm{p}<0.001)$. These associations remained significant when adjusting for BMI decreases $\geq 2 \% \quad(p=0.04$ for PFS and $\mathrm{p}=0.001$ for $\mathrm{OS}$ ), when analyzing BMI trend as a continuous variable $(p=0.01$ for PFS and $p<0.001$ for OS $)$, and when excluding patients who met criteria for cachexia $(\mathrm{p}=0.03$ for PFS and $\mathrm{p}=0.003$ for OS) (table 3).
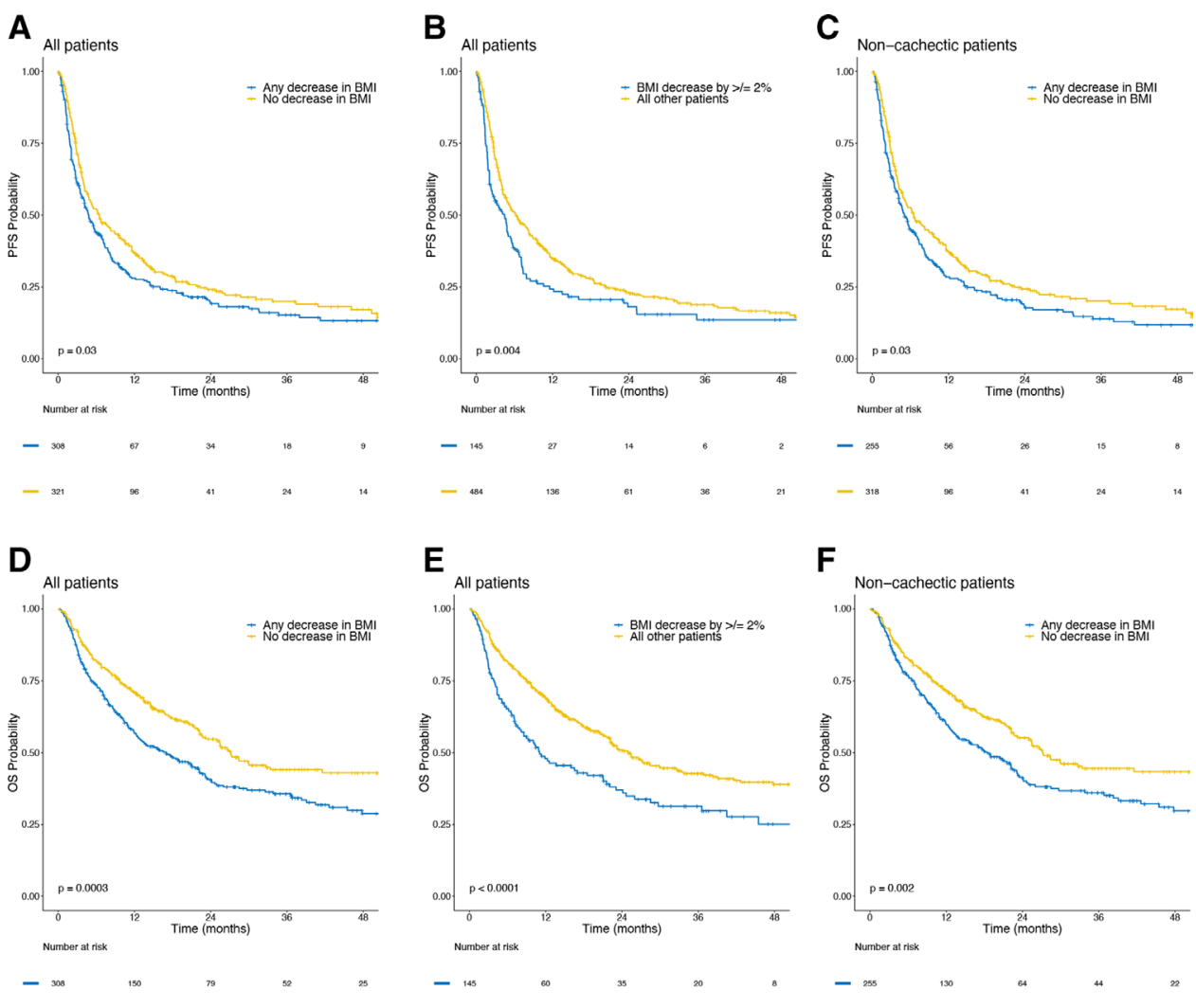

Figure 1 Kaplan-Meier curves showing progression-free survival (PFS) and overall survival (OS) by pretreatment body mass index (BMI) trends. PFS in (A) patients with any vs no decrease in pretreatment BMI among all patients, (B) BMI decrease of $\geq 2 \%$ vs all other patients and $(C)$ any vs no decrease in pretreatment BMI in patients who did not meet criteria for cachexia. OS in (D) patients with any vs no decrease in pretreatment BMI among all patients, (E) BMI decrease of $\geq 2 \%$ vs all other patients and $(F)$ any vs no decrease in pretreatment BMI in patients who did not meet criteria for cachexia. 
Table 3 Cox proportional-hazards regression: multivariable analyses

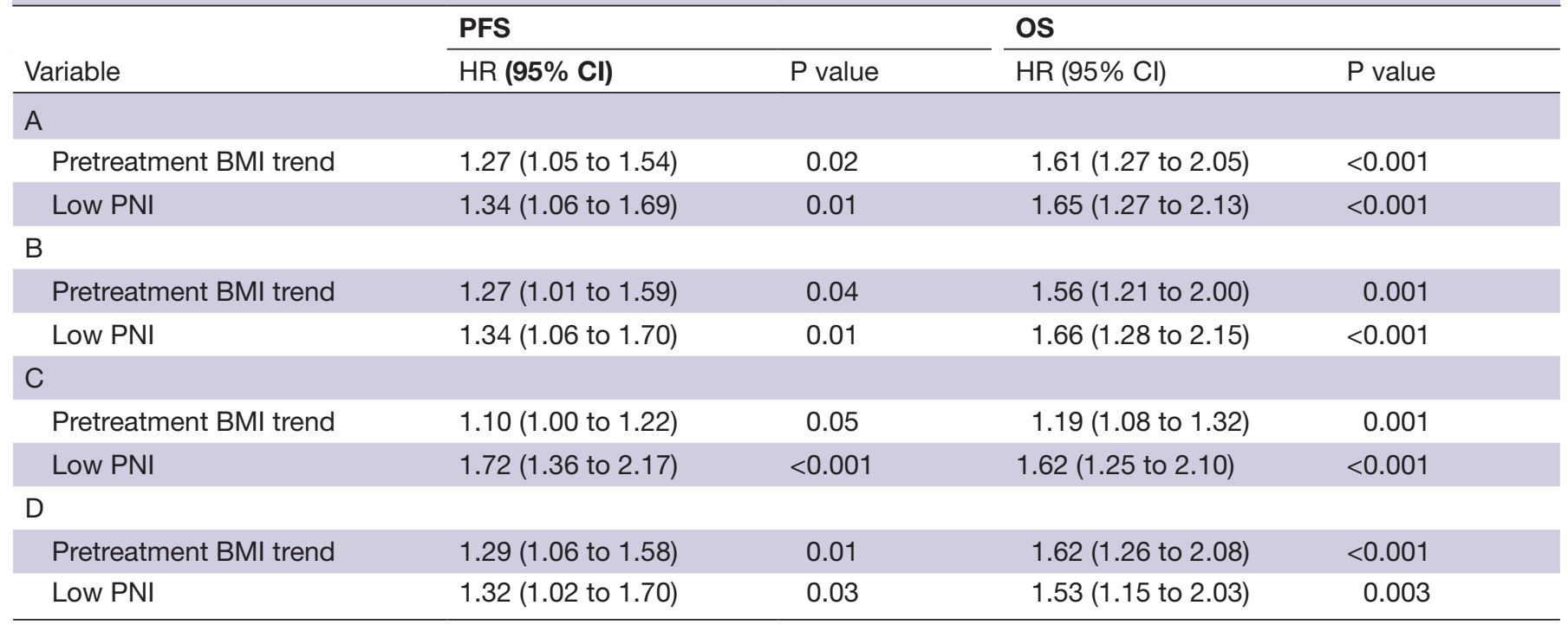

(A) All patients; any decrease in pretreatment BMI (reference: no decrease). (B) All patients; BMI decrease $\geq 2 \%$ (reference: all other patients). (C) All patients; pretreatment BMI change as a continuous variable. (D) Non-cachectic patients; any decrease in pretreatment BMI (reference: no decrease).

OS, overall survival; PFS, progression-free survival; PNI, prognostic nutritional index.

\section{Association of baseline BMI category with immunotherapy response}

There was a moderate but insignificant association between baseline BMI category (normal, overweight or obese) and best overall response $(p=0.11)$, ORR $(p=0.08)$ and disease control rate $(\mathrm{p}=0.14)$. In univariable analyses, baseline BMI category was not associated with PFS $(\mathrm{p}=0.19)$ (online supplemental figure 2 ). In multivariable analyses, there was no significant association between PFS and being overweight $(\mathrm{p}=0.10)$ or obese $(\mathrm{p}=0.56)$. This was also the case when analyzing baseline BMI as a continuous variable (HR 1.01; 95\% CI 0.99 to $1.03 ; \mathrm{p}=0.33$ ). In univariable analyses, baseline BMI category was significantly associated with OS $(\mathrm{p}=0.04)$ (online supplemental figure 2). However, in multivariable analyses, this association was not significant for either overweight patients $(\mathrm{p}=0.97)$ or patients with obesity $(\mathrm{p}=0.45)$ (online supplemental table 1). The association remained insignificant when analyzing baseline BMI as a continuous variable (HR 0.99; $95 \%$ CI 0.96 to $1.02 ; \mathrm{p}=0.38$ ). In a subset analysis of patients with stage 4 disease, there was no significant association between baseline BMI category and PFS or OS in univariable analyses $(\mathrm{p}=0.44$ and $\mathrm{p}=0.19$, respectively). The results of the multivariable analyses are shown

\begin{tabular}{|c|c|c|c|}
\hline Outcome & Low $(n=193)$ & Normal $(n=436)$ & $P$ value \\
\hline Best overall response-no. (\%) & & & 0.0004 \\
\hline Complete response (CR) & $12(6.2)$ & $56(12.8)$ & \\
\hline Partial response (PR) & $32(16.6)$ & $107(24.5)$ & \\
\hline Stable disease (SD) & $29(15.0)$ & 85 (19.5) & \\
\hline Progressive disease (PD) & $116(60.1)$ & $181(41.5)$ & \\
\hline Could not be evaluated & $4(2.1)$ & $7(1.6)$ & \\
\hline Objective response rate ${ }^{\star}$ & & & 0.0005 \\
\hline No. (\%) & $44(22.8)$ & $163(37.4)$ & \\
\hline $95 \% \mathrm{Cl} \dagger$ & 17.1 to 29.4 & 32.8 to 42.1 & \\
\hline Disease control rateł & & & $<0.0001$ \\
\hline No. $(\%)$ & $73(37.8)$ & $248(56.9)$ & \\
\hline $95 \% \mathrm{Cl} \dagger$ & 31.0 to 45.1 & 52.1 to 61.6 & \\
\hline
\end{tabular}

${ }^{*}$ Objective response rate was defined as the percentage of patients who had CR or PR.

†The $95 \%$ Cls were calculated using the Clopper-Pearson method.

$\ddagger$ Disease control rate was defined as the percentage of patients who had CR, PR or SD. 
A

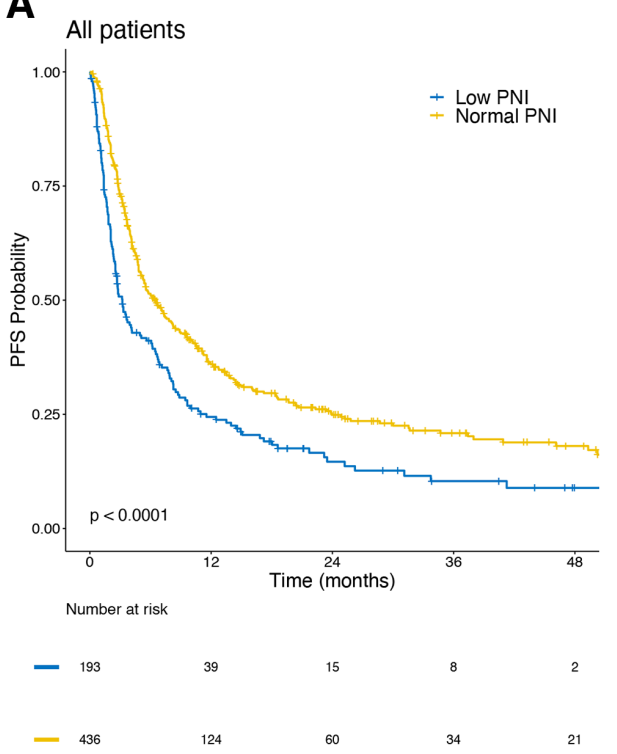

C

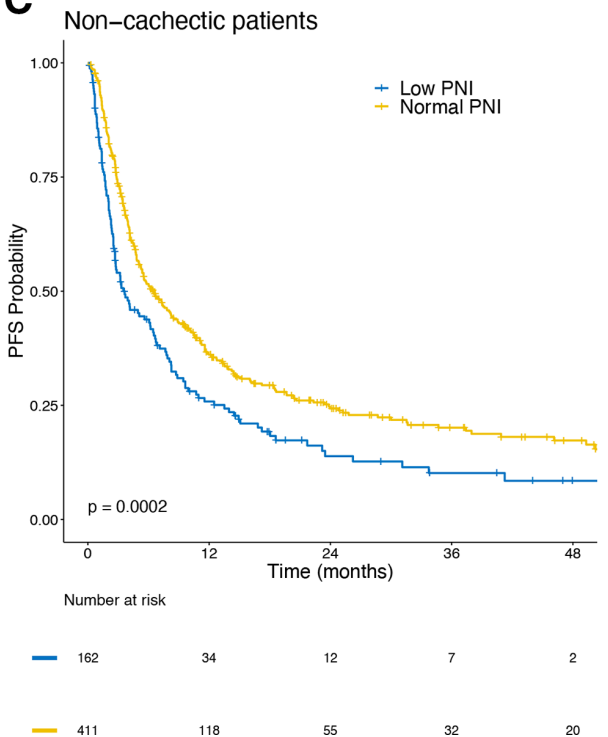

B

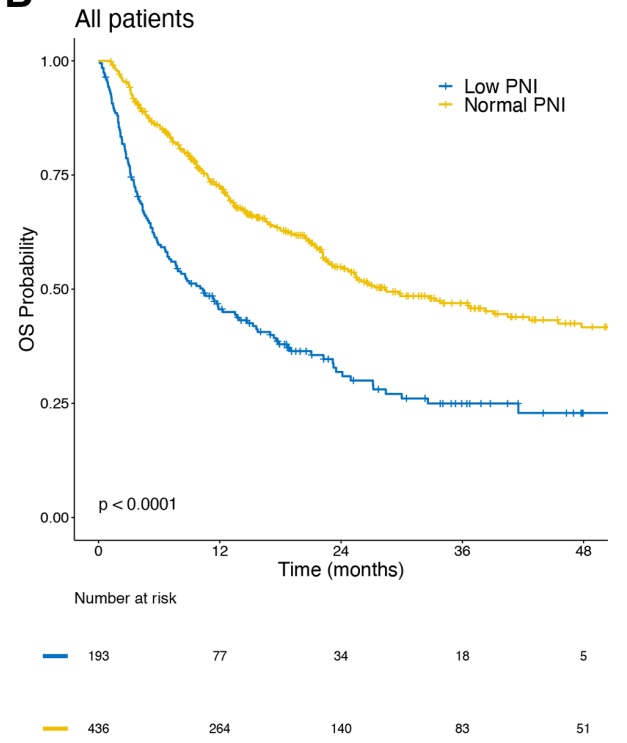

D

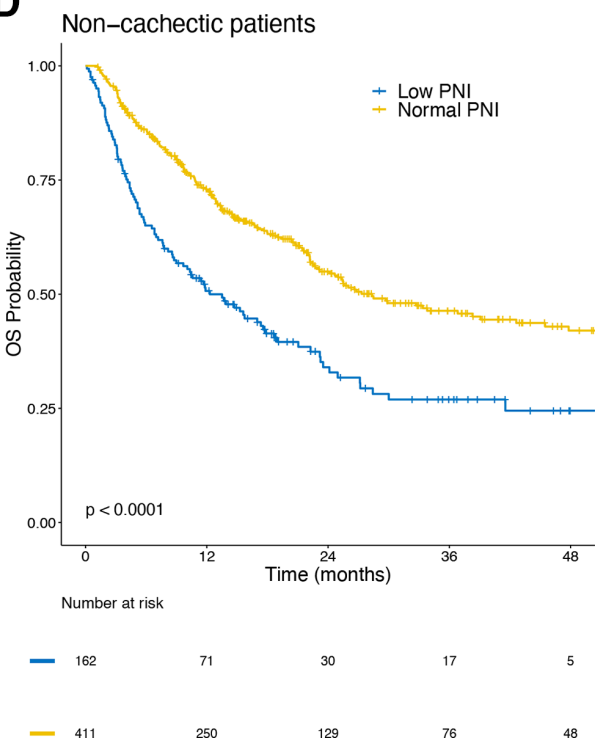

Figure 2 Kaplan-Meier curves showing progression-free survival (PFS) and overall survival (OS) by baseline prognostic nutritional index (PNI). PFS in (A) patients with low vs normal PNI among all patients, and (C) patients with low vs normal PNI in patients who did not meet criteria for cachexia. OS in (B) patients with low vs normal PNI among all patients, and (D) patients with low vs normal PNI in patients who did not meet criteria for cachexia.

in online supplemental table 2 and show no significant association. In a subset analysis of patients with only lung cancer, there was no significant association between baseline BMI and PFS or OS in the univariable analysis $(p=0.86$ and $p=0.73$, respectively). The results of the multivariable analysis are shown in online supplemental table 3 and show no significant associations.

When we compared patients with normal baseline BMI $(<25)$ with those with elevated baseline BMI $(\geq 25)$, we found that patients with elevated BMI had significantly better ORR (36.2\% (95\% CI 31.4 to 41.3 ) vs $27.8 \%$ (95\% CI 22.3 to 33.8$) ; \mathrm{p}=0.04)$. However, there was no significant difference in BOR $(\mathrm{p}=0.14)$. There was also no significant difference in DCR. Patients with elevated BMI had a DCR of $51.9 \%$ (95\% CI 46.8 to 57.1 ) while patients with normal BMI had a DCR of $49.6 \%$ (95\% CI 43.2 to 56.0 ) $(p=0.62)$. The association with PFS was insignificant in both univariable and multivariable analyses $(p=0.20$ and $\mathrm{p}=0.17$, respectively). In the univariable analysis, patients with normal BMI had significantly worse OS than patients with elevated BMI $(p=0.02)$. However, this relationship was not significant in the multivariable analysis $(\mathrm{p}=0.71)$.

\section{DISCUSSION}

In this study of patients with advanced cancer who received immune checkpoint inhibition, we tested the association between response to immunotherapy and 
three different nutritional indices: BMI changes prior to the start of treatment, baseline BMI category and baseline PNI. We observed that patients whose BMI decreased in the 15-45 days before treatment initiation suffered worse treatment outcomes, as did individuals with a low baseline PNI. There were no significant associations between baseline BMI category and ICI response. Our findings demonstrate two important points. First, pretreatment malnutrition, which is a modifiable variable, portended worse outcomes following checkpoint blockade independent of all possible confounding factors. Second, dynamic BMI trajectories, rather than static measurements, correlated with immunotherapy response. Taken together, these results underscore the importance of optimizing nutritional status as patients prepare for and receive ICI, and highlight the potential value of using BMI trends rather than baseline BMI category to characterize nutritional state.

The predictive value of nutritional status for ICI efficacy is not well-established, which is in contrast with the plethora of data supporting the association between malnutrition and overall survival. ${ }^{21-23}$ Only one recent study reported that patients with non-small cell lung cancer (NSCLC) with a PNI $\leq 45.5$ had significantly worse ORR, DCR and PFS as compared with patients with PNI $>45.5 .{ }^{24}$ Yet, there are several reasons that poor nutritional status could impair ICI effectiveness. First, hypercatabolic activity in response to malnutrition could lead to accelerated clearance of monoclonal antibodies, which are primarily eliminated via catabolic degradation. ${ }^{25}$ In fact, one group showed that decreased OS in patients with higher pembrolizumab clearance coincided with cancer cachexia, which speaks to the potential negative impact of elevated protein turnover. ${ }^{26}$ Second, chronic inflammation associated with malnutrition could paradoxically suppress activation of the adaptive immune system. Recent preclinical data show that heightened levels of the proinflammatory cytokine interleukin-6 (IL-6) induce systemic glucocorticoid release. ${ }^{27}$ This increased basal expression of endogenous steroids might dampen immune cell activity and consequently abrogate checkpoint inhibitor efficacy, as has been seen following the administration of exogenous steroids. ${ }^{28}$

Going forward, it will be important to determine whether and to what degree the optimization of nutritional status augments ICI response. Multiple prior studies, although not all, have shown that nutritional interventions are associated with improved survival in patients undergoing chemotherapy and radiation therapy, which suggests that dietary support can potentially yield meaningful clinical change. ${ }^{29-32}$ However, evidence suggests that the benefits are limited to patients at earlier stages of malnutrition as opposed to individuals with cancer cachexia, which is characterized by impaired anabolic signaling. ${ }^{33} 34$ In our study, we found that the subset of patients who were malnourished but did not meet criteria for cachexia still had worse treatment outcomes than patients who were not malnourished. Since these individuals are in the critical period of reversible malnutrition, they stand to benefit most from nutritional support and should be referred for dietary consultation. ${ }^{19} 35$ As expected, there is no catch-all strategy to managing weight loss and malnutrition in patients with cancer. Instead, it requires a multifaceted approach that includes but is not limited to regular screening, nutrition counseling, treatment of any symptoms that might impair food intake, prescription of oral nutritional supplements and possibly the use of pharmacological agents to improve appetite. ${ }^{36}$

It is noteworthy that pretreatment BMI trends, but not baseline BMI category, correlated with ICI response in this cohort of patients. We suspect that this is because longitudinal measurements capture dynamic changes in body composition and therefore more accurately reflect nutritional status than static measurements. Our findings call into question the predictive utility of baseline BMI. In fact, the extant literature already presents a mixed picture of pretreatment BMI category as a marker of response. For instance, some groups show that there is a linear relationship between increasing BMI and improving outcomes, while others report a U-shaped pattern, in which overweight but not obese patients exhibit mortality benefits. Additionally, there are discrepancies in the end points purportedly associated with BMI. Rogado et al found higher ORR and improved PFS in excess weight patients, whereas Richteg et al reported better ORR but no differences in PFS or OS. ${ }^{67}$ We evaluated multiple different clinical end points but could find no association between baseline BMI category and response to immunotherapy.

We showed that change in pretreatment BMI was associated with immunotherapy treatment outcomes when analyzed as either a categorical (decrease vs no decrease) or continuous variable. This suggests that even minor amounts of weight loss could have clinical implications for candidates of checkpoint blockade. That said, small decreases in BMI do not necessarily represent a true worsening of clinical status. There are a number of potential confounders including differences in measurement or intentional weight loss. Future studies should employ uniform protocols for anthropometric assessments. Since that was not possible for our study, we attempted to account for these cofounders by selectively analyzing the subset of patients who suffered $\geq 2 \%$ decrease in pretreatment BMI. We found that these individuals had significantly worse treatment outcomes following checkpoint blockade, which reinforces the validity of clinically significant decreases in BMI as a marker of response.

There are several limitations to this study. First, the PNI does not distinguish nutritional status from systemic inflammation. This is because inflammatory cytokines such as IL-6 regulate hepatocyte production of albumin, so hypoalbuminemia can be attributed to malnutrition, inflammation or both. ${ }^{12}$ Measuring $\mathrm{C}$ reactive protein and IL-6 levels could help determine whether and to what degree low PNI is due to systemic inflammation, however, we did not have these data available for analysis. In a similar vein, reporting prealbumin and transferrin levels 
could inform whether hypoalbuminemia reflects malnutrition. Albumin has a half-life of 20 days, but prealbumin and transferrin have shorter half-lives (2-3 and 10 days, respectively) and would provide a more comprehensive and up-to-date assessment of nutritional status. ${ }^{38}$ There are other prognostic scoring systems that can be used to select patients for treatment such as the Royal Marsden Hospital (RMH) and Gustave Roussy Immune (GRIm) Scores. ${ }^{39} 40$ The GRIm-Score includes neutrophil-tolymphocyte ratio and therefore has the potential to be more clinically relevant than PNI, which only incorporates the quantity of lymphocytes. However, both of these scoring systems include lactate dehydrogenase (LDH), and we only had pretreatment LDH values for a subset of our cohort, so we could not compare their predictive utility to that of the PNI. Finally, the diagnostic criteria for cachexia include weight loss $\geq 5 \%$ over the past 6 months, but we did not have information regarding patient weight this far before starting immunotherapy. Based on the available data, we assessed weight changes in the 15-45 days prior to treatment initiation. It is therefore possible that we did not identify all patients in the cohort who meet criteria for cachexia. Future studies need to include long-term weight trends starting at least 6 months before therapy in order to more precisely determine whether precachexia is associated with worse ICI response and whether outcomes improve following nutritional support in these patients.

In conclusion, we show that pretreatment BMI changes and baseline PNI are associated with immunotherapy response in patients with advanced cancer. These indicators of malnutrition are calculated using information that is readily available through routine standard of care and thus have potential for clinical implementation. Our findings need to be replicated in prospective studies in conjunction with other nutritional intake metrics, anthropometric measurements and laboratory markers in order to further elucidate the links between nutritional status and immunotherapy response. It is equally important to determine whether optimizing pretreatment nutritional status leads to improvement in clinical outcomes following checkpoint blockade.

Correction notice This paper has been updated to amend funder details.

Contributors Concept and design: PJ, AS, DD, 10. Acquisition, analysis or interpretation of data: all authors. Statistical analysis: PJ, YQ and JZ. Drafting and revision of manuscript: all authors. Supervision: 10.

Funding This work was supported by the NYU Melanoma Spore (P50CA225450) and the Laura and Isaac Perlmutter Cancer Center Support Grant (P30CA016087)

Competing interests None declared.

Patient consent for publication Not required.

Ethics approval This research is IRB approved (IRB \#10362 and IRB \#S1600122). All patients provided written informed consent to participate in this study.

Provenance and peer review Not commissioned; externally peer reviewed.

Data availability statement Data are available on reasonable request. Data are available from the authors on request but may require data transfer agreements. No personalized health information will be shared.
Supplemental material This content has been supplied by the author(s). It has not been vetted by BMJ Publishing Group Limited (BMJ) and may not have been peer-reviewed. Any opinions or recommendations discussed are solely those of the author(s) and are not endorsed by BMJ. BMJ disclaims all liability and responsibility arising from any reliance placed on the content. Where the content includes any translated material, BMJ does not warrant the accuracy and reliability of the translations (including but not limited to local regulations, clinical guidelines, terminology, drug names and drug dosages), and is not responsible for any error and/or omissions arising from translation and adaptation or otherwise.

Open access This is an open access article distributed in accordance with the Creative Commons Attribution Non Commercial (CC BY-NC 4.0) license, which permits others to distribute, remix, adapt, build upon this work non-commercially, and license their derivative works on different terms, provided the original work is properly cited, appropriate credit is given, any changes made indicated, and the use is non-commercial. See http://creativecommons.org/licenses/by-nc/4.0/.

\section{ORCID iD}

Paul Johannet http://orcid.org/0000-0002-9264-8327

\section{REFERENCES}

1 Hodi FS, O'Day SJ, McDermott DF, et al. Improved survival with ipilimumab in patients with metastatic melanoma. $N$ Engl J Med 2010;363:711-23.

2 Reck M, Rodríguez-Abreu D, Robinson AG, et al. Pembrolizumab versus chemotherapy for PD-L1-positive non-small-cell lung cancer. N Engl J Med 2016;375:1823-33.

3 Motzer RJ, Tannir NM, McDermott DF, et al. Nivolumab plus ipilimumab versus sunitinib in advanced renal-cell carcinoma. $N$ Engl J Med 2018;378:1277-90.

4 Michot JM, Bigenwald C, Champiat S, et al. Immune-Related adverse events with immune checkpoint blockade: a comprehensive review. Eur J Cancer 2016;54:139-48.

5 Naik GS, Waikar SS, Johnson AEW, et al. Complex inter-relationship of body mass index, gender and serum creatinine on survival: exploring the obesity paradox in melanoma patients treated with checkpoint inhibition. J Immunother Cancer 2019;7:89.

6 Richtig G, Hoeller C, Wolf M, et al. Body mass index may predict the response to ipilimumab in metastatic melanoma: an observational multi-centre study. PLoS One 2018;13:e0204729.

7 Kichenadasse G, Miners JO, Mangoni AA, et al. Association between body mass index and overall survival with immune checkpoint inhibitor therapy for advanced non-small cell lung cancer. JAMA Oncol 2020;6:512.

8 Cortellini A, Bersanelli M, Buti S, et al. A multicenter study of body mass index in cancer patients treated with anti-PD-1/PD-L1 immune checkpoint inhibitors: when overweight becomes favorable. $J$ Immunother Cancer 2019;7:57.

9 McQuade JL, Daniel CR, Hess KR, et al. Association of bodymass index and outcomes in patients with metastatic melanoma treated with targeted therapy, immunotherapy, or chemotherapy: a retrospective, multicohort analysis. Lancet Oncol 2018;19:310-22.

10 Naik A, Monjazeb AM, Decock J. The obesity paradox in cancer, tumor immunology, and immunotherapy: potential therapeutic implications in triple negative breast cancer. Front Immunol 2019;10:10.

11 Donnelly D, Bajaj S, Yu J, et al. The complex relationship between body mass index and response to immune checkpoint inhibition in metastatic melanoma patients. J Immunother Cancer 2019;7:222.

12 Mirili C, Yılmaz A, Demirkan S, et al. Clinical significance of prognostic nutritional index (PNI) in malignant melanoma. Int $\mathrm{J}$ Clin Oncol 2019;24:1301-10.

13 Hong $X$, Cui B, Wang M, et al. Systemic immune-inflammation index, based on platelet counts and neutrophil-lymphocyte ratio, is useful for predicting prognosis in small cell lung cancer. Tohoku J Exp Med 2015;236:297-304.

14 Kinoshita A, Onoda H, Imai N, et al. Comparison of the prognostic value of inflammation-based prognostic scores in patients with hepatocellular carcinoma. Br J Cancer 2012;107:988-93.

15 Hong S, Zhou T, Fang W, et al. The prognostic nutritional index (PNI) predicts overall survival of small-cell lung cancer patients. Tumour Biol 2015;36:3389-97.

16 Shimizu T, Taniguchi K, Asakuma M, et al. Lymphocyte-To-Monocyte ratio and prognostic nutritional index predict poor prognosis in patients on chemotherapy for unresectable pancreatic cancer. Anticancer Res 2019;39:2169-76.

17 Minami S, Ogata Y, Ihara S, et al. Pretreatment Glasgow prognostic score and prognostic nutritional index predict overall survival 
of patients with advanced small cell lung cancer. Lung Cancer 2017:8:249-57.

18 Chen Q-J, Qu H-J, Li D-Z, et al. Prognostic nutritional index predicts clinical outcome in patients with acute ST-segment elevation myocardial infarction undergoing primary percutaneous coronary intervention. Sci Rep 2017;7:3285.

19 Fearon K, Strasser F, Anker SD, et al. Definition and classification of cancer cachexia: an international consensus. Lancet Oncol 2011;12:489-95.

20 Liu J, Lichtenberg T, Hoadley KA, et al. An integrated TCGA pancancer clinical data resource to drive high-quality survival outcome analytics. Cell 2018;173:400-16.

21 Bullock AF, Greenley SL, McKenzie GAG, et al. Relationship between markers of malnutrition and clinical outcomes in older adults with cancer: systematic review, narrative synthesis and meta-analysis. Eur J Clin Nutr 202010.1038/s41430-020-0629-0. [Epub ahead of print: 04 May 2020].

22 Lee C-S, Devoe CE, Zhu X, et al. Pretreatment nutritional status and response to checkpoint inhibitors in lung cancer. Lung Cancer Manag 2020;9:LMT31.

23 Cortellini A, Bozzetti F, Palumbo P, et al. Weighing the role of skeletal muscle mass and muscle density in cancer patients receiving PD-1/ PD-L1 checkpoint inhibitors: a multicenter real-life study. Sci Rep 2020;10:1456

24 Shoji F, Takeoka H, Kozuma Y, et al. Pretreatment prognostic nutritional index as a novel biomarker in non-small cell lung cancer patients treated with immune checkpoint inhibitors. Lung Cancer 2019;136:45-51.

25 Ryman JT, Meibohm B. Pharmacokinetics of monoclonal antibodies. CPT Pharmacometrics Syst Pharmacol 2017;6:576-88.

26 Turner DC, Kondic AG, Anderson KM, et al. Pembrolizumab exposure-response assessments challenged by association of cancer cachexia and catabolic clearance. Clin Cancer Res 2018;24:5841-9.

27 Flint TR, Fearon DT, Janowitz T. Connecting the metabolic and immune responses to cancer. Trends Mol Med 2017;23:451-64.

28 Giles AJ, Hutchinson M-KND, Sonnemann HM, et al. Dexamethasone-Induced immunosuppression: mechanisms and implications for immunotherapy. J Immunother Cancer 2018;6:51.
29 Ravasco P, Monteiro-Grillo I, Camilo M. Individualized nutrition intervention is of major benefit to colorectal cancer patients: longterm follow-up of a randomized controlled trial of nutritional therapy. Am J Clin Nutr 2012;96:1346-53.

30 Qiu M, Zhou Y-xin, Jin Y, et al. Nutrition support can bring survival benefit to high nutrition risk gastric cancer patients who received chemotherapy. Support Care Cancer 2015;23:1933-9.

31 Bourdel-Marchasson I, Blanc-Bisson C, Doussau A, et al. Nutritional advice in older patients at risk of malnutrition during treatment for chemotherapy: a two-year randomized controlled trial. PLoS One 2014;9:e108687.

32 Baldwin C, Spiro A, Ahern R, et al. Oral nutritional interventions in malnourished patients with cancer: a systematic review and metaanalysis. J Natl Cancer Inst 2012;104:371-85.

33 Prado CM, Sawyer MB, Ghosh S, et al. Central tenet of cancer cachexia therapy: do patients with advanced cancer have exploitable anabolic potential? Am J Clin Nutr 2013;98:1012-9.

34 Bowen TS, Schuler G, Adams V. Skeletal muscle wasting in cachexia and sarcopenia: molecular pathophysiology and impact of exercise training. J Cachexia Sarcopenia Muscle 2015;6:197-207.

35 Dev R. Measuring cachexia-diagnostic criteria. Ann Palliat Med 2019;8:24-32.

36 Arends J, Bachmann P, Baracos V, et al. ESPEN guidelines on nutrition in cancer patients. Clin Nutr 2017;36:11-48.

37 Rogado J, Romero-Laorden N, Sanchez-Torres JM, et al. Effect of excess weight and immune-related adverse events on the efficacy of cancer immunotherapy with anti-PD-1 antibodies. Oncoimmunology 2020;9:e1751548.

38 Bharadwaj S, Ginoya S, Tandon P, et al. Malnutrition: laboratory markers vs nutritional assessment. Gastroenterol Rep 2016;4:gow013-280.

39 Bigot F, Castanon E, Baldini C, et al. Prospective validation of a prognostic score for patients in immunotherapy phase I trials: the Gustave Roussy immune score (GRIm-Score). Eur J Cancer 2017;84:212-8

40 Arkenau H-T, Barriuso J, Olmos D, et al. Prospective validation of a prognostic score to improve patient selection for oncology phase I trials. J Clin Oncol 2009;27:2692-6. 
Correction: Baseline prognostic nutritional index and changes in pretreatment body mass index associate with immunotherapy response in patients with advanced cancer

Johannet P, Sawyers A, Qian Y, et al. Baseline prognostic nutritional index and changes in pretreatment body mass index associate with immunotherapy response in patients with advanced cancer. J Immunother Cancer 2020;8:e01674. doi: 10.1136/jitc-2020001674

This paper has been updated to amend funder details.

Open access This is an open access article distributed in accordance with the Creative Commons Attribution Non Commercial (CC BY-NC 4.0) license, which permits others to distribute, remix, adapt, build upon this work non-commercially, and license their derivative works on different terms, provided the original work is properly cited, appropriate credit is given, any changes made indicated, and the use is non-commercial. See http://creativecommons.org/licenses/by-nc/4.0/.

(C) Author(s) (or their employer(s)) 2021. Re-use permitted under CC BY-NC. No commercial re-use. See rights and permissions. Published by BMJ.

J Immunother Cancer 2021;9:e001674corr1. doi:10.1136/jitc-2020-001674corr1

A) Check for updates 